\title{
Improving Diffusion-Based Molecular Communication with Unanchored Enzymes
}

\author{
Adam Noel ${ }^{\star}$, Karen Cheung, and Robert Schober \\ Department of Electrical and Computer Engineering \\ University of British Columbia, Vancouver BC, Canada \\ adamn@ece.ubc.ca
}

\begin{abstract}
In this paper, we propose adding enzymes to the propagation environment of a diffusive molecular communication system as a strategy for mitigating intersymbol interference. The enzymes form reaction intermediates with information molecules and then degrade them so that they have a smaller chance of interfering with future transmissions. We present the reaction-diffusion dynamics of this proposed system and derive a lower bound expression for the expected number of molecules observed at the receiver. We justify a particle-based simulation framework, and present simulation results that show both the accuracy of our expression and the potential for enzymes to improve communication performance.
\end{abstract}

Key words: Molecular communication, reaction-diffusion system, intersymbol interference, nanonetwork.

\section{Introduction}

Molecular communication is the use of molecules emitted by a transmitter into its surrounding environment to carry information to an intended receiver. This strategy has recently emerged as a popular choice for the design of new communication networks where devices with nanoscale components need to communicate with each other, i.e., nanonetworks. Molecular communication is suitable because its inherent biocompatibility can facilitate implementation inside of a living organism; many mechanisms in cells, organisms, and subcellular structures already rely on the transmission of molecules for communication, as described in [1, Ch. 16]. It is envisioned, as in [2, 3, that by using bio-hybrid components (such as synthesized proteins or genetically-modified cells), we can take advantage of these mechanisms for a range of applications that can include health monitoring, targeted drug delivery, and nanotechnology in general.

The design of molecular communication systems should reflect both the limited capabilities of small individual transceivers and the physical environment in which they operate. The state-of-the-art has only begun to take advantage of

\footnotetext{
* The first author was supported by the Natural Sciences and Engineering Research Council of Canada, and a Walter C. Sumner Memorial Fellowship. Computing resources were provided by WestGrid and Compute/Calcul Canada.
} 
the unique characteristics of molecular communication systems and their operational environments. The simplest and arguably most popular molecular communication scheme proposed has been communication via diffusion. Diffusion is a naturally-occurring process where free molecules tend to disperse through a medium over time. Diffusion requires no added energy and can be very fast over short distances; bacterial cells, many of which are on the order of one micron in diameter, can rely on diffusion for all of their internal transport requirements; see [4, Ch. 4]. By adopting diffusion, network designers do not need to worry about the development of the infrastructure required for active methods such as the molecular motors described in [5].

The major drawbacks of using diffusion are the need for a large number of information molecules to send a single message, long propagation times over larger distances, and the intersymbol interference (ISI) due to molecules taking a long time to diffuse away. Fortunately, biological systems commonly store large numbers of molecules for release at specific instances, such as the storage of Calcium ions in cellular vesicles until they are needed for signalling or secretion, as described in [1, Ch. 16]. Thus, delay and ISI become the performance bottlenecks. Strategies in the literature for mitigating ISI have been limited to making the transmitter wait sufficiently long for the presence of previouslyemitted molecules to become negligible, as in [6] 8. The primary drawback of this strategy is a reduced transmission rate.

We propose adding reactive molecules to the propagation environment to significantly decrease the ISI in a molecular communication link when a single type of information molecule is used. The reactive molecules transform the information molecules so that they are no longer recognized by the receiver. If using chemical reactants, then they must be provided in stoichiometric excess relative to the information molecules, otherwise their capacity to transform those molecules may be limited over time. However, a catalyst lowers the activation energy for a specific biochemical reaction but does not appear in the stoichiometric expression of the complete reaction so (unlike a reactant) is not consumed.

An enzyme is a biomolecule that acts as a catalyst, often by providing an active site (a groove or pocket) that encourages a particular molecular conformation; see [1, Ch. 3]. Compared to catalysts in general, enzymes can have the advantage of very high selectivity for their substrates. Thus, we are specifically interested in enzymes as reactive molecules because a single enzyme can be recycled to react many times. Enzymes play a key role in many essential biochemical reactions. For example, acetylcholinesterase is an enzyme present in the neuromuscular junction that hydrolyzes diffusing acetylcholine to prevent continued activation in the post-synaptic membrane because the receptor in the membrane does not recognize acetate or choline, as described in [4, Ch. 12]. Acetylcholine is called the substrate for acetylcholinesterase. The physical environment of the neuromuscular junction is referred to as a reaction-diffusion system because reaction and diffusion can take place simultaneously. From a purely communications perspective, the enzyme in this example is reducing the ISI of the substrate. 
There are many potential benefits for using enzymes to aid in developing new molecular communication systems. The reduction in ISI would enable transmitters to release molecules more often, simultaneously increasing the data rate and decreasing the probability of erroneous transmission. There would also be less interference from neighbouring communication links, so independent senderreceiver pairs could be placed closer together than in an environment dominated by diffusion alone. These gains can be achieved with no additional complexity at the sender or receiver, which is a very useful benefit for the case of individual nanomachines with limited computational capabilities. The enzymatic reaction mechanism could also be coupled to a mechanism that regenerates information molecules once they are degraded so that they are returned to the sender for future use (as is the case for acetylcholinesterase). Of course, it is necessary to select an enzyme-substrate pair that would not otherwise damage the environment where the nanomachines are in operation.

Most existing work in molecular communications, including [9] and [10], have considered enzymes only at the receiver as part of the reception mechanism. In these cases, the ability for the enzymes to mitigate ISI is limited. Two works that have considered information molecules reacting in the propagation environment are [11,12]. In [11, the spontaneous destruction and duplication of information molecules are treated as noise sources, whereas in [12, information molecules undergo exponential decay in an attempt to mitigate ISI. Papers that have considered reaction-diffusion systems with enzymes in the propagation environment from a biological perspective, such as [13] and [14] for acetylcholinesterase, have focussed on providing an accurate simulation model for specific biological processes with a particular physical layout and not the manipulation of parameters for the design of new communication systems.

In this paper, we present a model for the analysis of diffusion-based communication systems with enzymes that are present throughout the entire propagation environment. We start with the fundamental dynamics of both diffusion and enzyme kinetics to derive a bound on the expected number of molecules within the volume of an isolated observer placed some distance from the transmitter. In this context, we assume that the reader has a communications background and is not familiar with reaction-diffusion dynamics. We justify a particle-based simulation framework to assess the accuracy of our analytical results, and show that adding enzymes drastically reduces the "tail" created by relying on diffusion alone.

The rest of this paper is organized as follows. In Section 2, we introduce our model for transmission between a single transmitter and receiver. This model is based on both reaction and diffusion. In Section 3 we derive the number of information molecules expected at the receiver. We present the simulation framework in Section 4 before giving numerical and simulation results in Section 5 . In Section 6 6 we present conclusions and discuss the on-going and future direction of our analysis.

Unless otherwise noted, we use meters (m) for distance, seconds ( $\mathrm{s}$ ) for time, and molecules per $\mathrm{m}^{3}$ for concentrations (concentrations are typically given in 


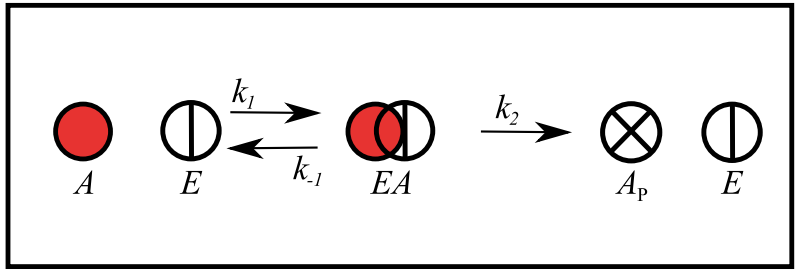

Fig. 1. The Michaelis-Menten reaction mechanism. Substrate molecule $A$ can react with enzyme molecule $E$ if they collide with sufficient energy and in the correct orientation. The reaction produces an intermediate $E A$ that can either return to its original constituents or degrade particle $A$ into $A_{P}$. The enzyme is not degraded by this process so it can react with multiple $A$ molecules. An $A$ molecule, once degraded, cannot be returned to its original state via this mechanism.

moles per litre, but molecules per $\mathrm{m}^{3}$ makes our analysis easier to follow by limiting the number of conversions).

\section{System Model}

We consider an unbounded 3-dimensional aqueous environment. There is a sender fixed at the origin, treated analytically as a point source but as a sphere in simulation. The receiver is a fixed spherical volume of radius $r_{o b s}$ and size $V_{o b s}$, centered at the point defined by $\overrightarrow{r_{0}}=\left\{x_{0}, y_{0}, z_{0}\right\}$. The receiver acts as a passive observer by not disturbing the diffusion of any molecules in the environment. This is not a strong assumption, since many small molecules are able to diffuse freely through cells and other objects if the molecules are non-polar or if there are protein channels specific to the molecules in the cell's plasma membrane; see [1, Ch. 12]. The immobility of both the sender and receiver is generally impractical at the nanoscale unless they are anchored to larger objects, but here we assume immobility for ease of analysis.

Before describing our communication process, we must overview the environment's chemical dynamics. There are three mobile species (types of molecules) in the system that we are interested in: $A$ molecules, $E$ molecules, and $E A$ molecules. The number of molecules of species $S$ is given by $N_{S}$ where $S \in$ $\{A, E, E A\}$. $A$ molecules are the information molecules that are released by the sender. These molecules have a natural degradation rate that is negligible over the time scale of interest, but they are able to act as substrates with enzyme $E$ molecules. We assume that $A$ and $E$ molecules react according to the following Michaelis-Menten reaction mechanism (which is generally accepted as the fundamental mechanism for enzymatic reactions; see [4, 15], and Fig. 11):

$$
\begin{aligned}
E+A & \stackrel{k_{1}}{\longrightarrow} E A, \\
& E A \stackrel{k_{-1}}{\longrightarrow} E+A, \\
& E A \stackrel{k_{2}}{\longrightarrow} E+A_{P},
\end{aligned}
$$


where $E A$ is the intermediate formed by the binding of an $A$ molecule to an enzyme molecule, $A_{P}$ is the degraded $A$ molecule, and $k_{1}, k_{-1}$, and $k_{2}$ are the reaction rates for the reactions as shown with units molecule ${ }^{-1} \mathrm{~m}^{3} \mathrm{~s}^{-1}, \mathrm{~s}^{-1}$, and $\mathrm{s}^{-1}$, respectively. We see that $A$ molecules are irreversibly degraded by reaction (3) while the enzymes are released intact so that they can participate in future reactions. We are not interested in the $A_{P}$ molecules once they are formed because they cannot participate in future reactions.

We assume that every molecule of each species $S$ diffuses independently of all other molecules, unless they are bound together. We assume that all free molecules are spherical in shape so that we can state that each molecule diffuses with diffusion constant $D_{S}$, found using the Einstein relation as [4, Eq. 4.16]

$$
D_{S}=\frac{k_{B} T}{6 \pi \eta R_{S}},
$$

where $k_{B}$ is the Boltzmann constant $\left(k_{B}=1.38 \times 10^{-23} \mathrm{~J} / \mathrm{K}\right), T$ is the temperature in kelvin, $\eta$ is the viscosity of the medium in which the particle is diffusing ( $\eta \approx 10^{-3} \mathrm{~kg} \mathrm{~m}^{-1} \mathrm{~s}^{-1}$ for water at room temperature), and $R_{S}$ is the molecule radius. Thus, the units for $D_{S}$ are $\mathrm{m}^{2} / \mathrm{s}$. The diffusion of a single molecule along one dimension has variance $2 D_{S} t$, where $t$ is the diffusing time [4, Eq. 4.6].

We note that reaction rate constants are experimentally measured for specific reactions under specific environmental conditions (i.e., temperature, $\mathrm{pH}$, etc.) using large populations of each reactant. By "large", we mean sufficiently large for the rate of change of species concentrations to be deterministic. Diffusion also becomes deterministic with sufficiently large populations. We are not interested in such large populations due to the size of our system. However, the rate constants also describe the stochastic affinity of reactions in single-molecule detail, as proven in [16. It is impossible to precisely predict where a specific molecule will diffuse and if or when it will react with other molecules, but the diffusion and reaction rate constants will be used to generate random variables when executing stochastic simulations of system behavior.

We can now describe the communication process. The sender emits impulses of $N_{A} A$ molecules, which is a common emission scheme in the molecular communication literature; see, for example, [6]. We deploy binary modulation with constant bit interval $T_{B}$, where $N_{A}$ molecules are released at the start of the interval for binary 1 and no molecules are released for binary 0 (there have been works studying the use of different $T_{B}$ s depending on the values of the current and previous bit, as in 8 , since, for example, consecutive 0s can be transmitted with less risk of intersymbol interference). $N_{E} E$ molecules are randomly (uniformly) distributed throughout a finite cubic volume $V_{\text {enz }}$ that includes both the sender (TX) and receiver (RX), as shown in Fig. 2. $V_{\text {enz }}$ is impermeable to $E$ molecules (so that we can simulate using a finite number of $E$ molecules) but not $A$ molecules (in simulation, we make $E A$ molecules decompose to their constituents if they hit the boundary). Therefore, the total concentration of the free and bound enzyme is constant. $V_{\text {enz }}$ is sufficiently large to assume that it is infinite in size, such that there would be negligible change in observations at the receiver if $V_{\text {enz }}$ were also impermeable to $A$ molecules. 


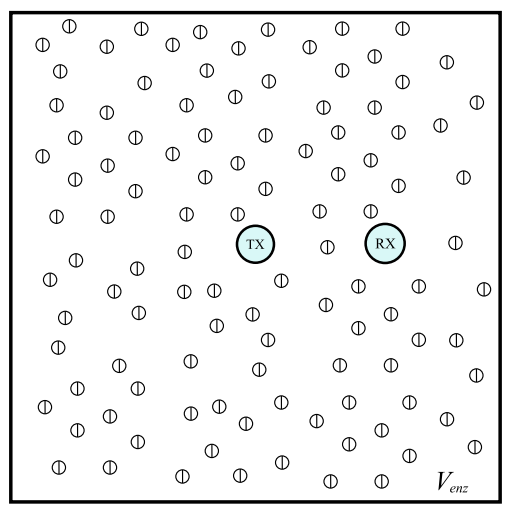

Fig. 2. The bounded space $V_{e n z}$ in 2-dimensions showing the initial uniform distribution of enzyme $E$. $V_{\text {enz }}$ inhibits the passage of $E$ so that the total concentration of free and bound $E$ remains constant. $A$ molecules can diffuse beyond $V_{\text {enz }}$.

The receiver counts the number of free (unbound) $A$ molecules that are within the receiver volume, without disturbing those molecules. For a practical biohybrid system, the $A$ molecules would need to bind to receptors on either the receiver surface or within the receiver's volume, but we assume perfect passive counting in order to focus on the propagation environment. We also assume that the degraded $A_{P}$ molecules were modified in such a way that they cannot be detected by the receiver, so $A_{P}$ molecules can be ignored.

\section{Observations at the Receiver}

Generally, the spatial-temporal behavior of the three mobile species can be described using a system of reaction-diffusion partial differential equations. Even though these equations are deterministic, we noted in Section 2 that they will enable stochastic simulation. In this section, we use the deterministic partial differential equations to derive the expected number of information molecules at the receiver.

\subsection{Diffusion Only}

For comparison, we first consider the dynamics when there is no enzyme present, i.e., $N_{E}=0$. So, we only consider the diffusion of $A$ molecules in the unbounded environment. By Fick's Second Law we have [4, Ch. 4]

$$
\frac{\partial C_{S}(\vec{r}, t)}{\partial t}=D_{S} \nabla^{2} C_{S}(\vec{r}, t)
$$

where $C_{S}(\vec{r}, t)$ is the point concentration of species $S$ at time $t$ and location $\vec{r}$. Closed-form analytical solutions for partial differential equations are not always 
possible and depend on the boundary conditions that are imposed. Here, we have no $E$ molecules, so there are also no $E A$ molecules, and we immediately have $C_{E}(\vec{r}, t)=C_{E A}(\vec{r}, t)=0 \forall \vec{r}, t$. Assuming that the $A$ molecules are released from the origin at $t=0$, we then have [4, Eq. 4.28]

$$
C_{A}(\vec{r}, t)=\frac{N_{A}}{\left(4 \pi D_{A} t\right)^{3 / 2}} \exp \left(\frac{-|\vec{r}|^{2}}{4 D_{A} t}\right) .
$$

Eq. (6) is the form that is typically used in molecular communications to describe the local concentration at the receiver; the receiver is assumed to be a point observer, as in [7,17, or the concentration throughout the receiver volume is assumed to be uniform and equal to that expected in the center, as in [6. Eq. (6) is the baseline against which we will evaluate our proposed system design.

It has been noted that Fick's second law violates the theory of special relativity, since there is no bound on how far a single particle can travel within a given time. A finite propagation speed can be added as a correction, as in [18, but we assume that Fick's second law is sufficiently accurate without this correction.

\subsection{Reaction-Diffusion}

We now include active enzymes in our analysis. If we write $C_{S}(\vec{r}, t)=C_{S}$, $S \in\{A, E, E A\}$, for compactness, then the general reaction-diffusion equation is [19, Eq. 8.12.1]

$$
\frac{\partial C_{S}}{\partial t}=D_{S} \nabla^{2} C_{S}+f\left(C_{S}, \vec{r}, t\right),
$$

where $f(\cdot)$ is the reaction term. Using the principles of chemical kinetics (see [15, Ch. 9]), we write the complete partial differential equations for the species in our system as

$$
\begin{aligned}
\frac{\partial C_{A}}{\partial t} & =D_{A} \nabla^{2} C_{A}-k_{1} C_{A} C_{E}+k_{-1} C_{E A}, \\
\frac{\partial C_{E}}{\partial t} & =D_{E} \nabla^{2} C_{E}-k_{1} C_{A} C_{E}+k_{-1} C_{E A}+k_{2} C_{E A}, \\
\frac{\partial C_{E A}}{\partial t} & =D_{A} \nabla^{2} C_{E A}+k_{1} C_{A} C_{E}-k_{-1} C_{E A}-k_{2} C_{E A} .
\end{aligned}
$$

This system of equations is highly coupled due to the reaction terms and has no closed-form analytical solution under our boundary conditions. We seek such a solution, so we must make some simplifying assumptions. We first note that the total concentration of enzyme, both free and bound to $A$, over the entire system is always constant $C_{E_{T o t}}=N_{E} / V_{e n z}$. A common next step for the Michaelis-Menten mechanism in (11)-(3) is to assume that the amount of $E A$ is constant, i.e., $\frac{\partial C_{E A}}{\partial t}=0$, in order to derive an expression for $C_{E A}$; see [15, Ch. 10] and its use when considering enzymes at the receiver in [10]. We will use a slightly different assumption to directly derive a lower bound expression. We assume that both $C_{E}$ and $C_{E A}$ are not time-varying, i.e., $C_{E}$ and $C_{E A}$ are both constants. It is then straightforward to show that, in our system, (8) has solution 


$$
C_{A} \approx \frac{N_{A}}{\left(4 \pi D_{A} t\right)^{3 / 2}} \exp \left(-k_{1} C_{E} t-\frac{|\vec{r}|^{2}}{4 D_{A} t}\right)+k_{-1} C_{E A} t,
$$

and we ignore (9) and (10). Next, we assume that the amount of $E A$ at any time is small, such that $k_{-1} C_{E A} \rightarrow 0$. If $C_{E A}$ is small, then we can approximate $C_{E}$ with its upper bound $C_{E_{T o t}}$. All concentrations and rate constants must be non-negative, so we can write the bound

$$
C_{A} \geq \frac{N_{A}}{\left(4 \pi D_{A} t\right)^{3 / 2}} \exp \left(-k_{1} C_{E_{T o t}} t-\frac{|\vec{r}|^{2}}{4 D_{A} t}\right),
$$

which is intuitively a lower bound because the actual degradation due to enzymes can be no more than if all enzymes were always unbound. In other words, (12) describes the point concentration of $A$ molecules as $k_{2} \rightarrow \infty$ and $k_{-1} \rightarrow 0$. A convenient property of this lower bound is that, while it loses accuracy as $E A$ is initially created $\left(C_{E}<C_{E_{T o t}}\right)$, it eventually improves with time as all $A$ molecules are degraded and none remain to bind with the enzyme $\left(C_{A}, C_{E A} \rightarrow 0\right.$, $C_{E} \rightarrow C_{E_{T o t}}$, as $\left.t \rightarrow \infty\right)$. We also note that, had we started with the $\frac{\partial C_{E A}}{\partial t}=0$ assumption, then we would have arrived at a similar expression to (12), where $k_{1}$ is replaced with $k_{1} k_{2} /\left(k_{-1}+k_{2}\right)$.

Eq. (12) can be directly compared with (6). The presence of enzyme results in an additional decaying exponential term. This decaying exponential is what will eliminate the "tail" that is observed under diffusion alone. It can be shown that adding enzymes will always lead to a faster degradation of $C_{A}$ from its maximum value for a given $\vec{r}$ than when not adding enzymes. Furthermore, the maximum value is achieved sooner when enzymes are present, but this value is smaller. These statements are apparent from the results in Section 5 and future work will prove these statements analytically.

We have already established that the receiver is able to count the number of free $A$ molecules that are within the receiver volume. Eqs. (12) and (6) give us the expected point concentrations with and without active enzymes, respectively. We can readily convert these concentrations to the expected number of observed $A$ molecules, $\overline{N_{A o b s}}(t)=C_{A}\left(\overrightarrow{r_{0}}, t\right) V_{o b s}$, if we assume that the concentration throughout the receiver is uniform, as in [6].

\section{Simulation Framework}

In the previous section, we derived (12) as a lower bound on the local concentration when enzymes are present throughout the propagation environment. We now require an appropriate simulation framework to evaluate the accuracy of (12). This framework will be used to perform stochastic simulations of the system of equations described by (8)-(10).

\subsection{Choice of Framework}

Commonly used stochastic reaction-diffusion simulation platforms can be placed into one of two categories. The first are subvolume-based methods, where the 
reaction environment is divided into one (if diffusion is ignored) or many wellstirred subvolumes. By well-stirred, it is meant that molecules in a specific subvolume are uniformly distributed throughout that subvolume, and that the velocities of those molecules follow the Boltzmann distribution; see [16. In other words, every subvolume should have more nonreactive molecular collisions than reactive collisions. Stochastic subvolume-based methods are based on the stochastic simulation algorithm, which generates random numbers to determine the time and type of the next reaction in the system; see [20. We note that these methods, though subvolume-based, still consider discrete species populations. However, the precise locations of individual molecules are not maintained, and diffusion is modeled as transitions of molecules between adjacent subvolumes; see [21].

The second category of simulation platforms use particle-based methods, where the precise locations of all individual molecules are known. Every free molecule diffuses independently along each dimension. These methods require a constant global time step $\Delta t$ and there is a separation in the simulation of reaction and diffusion; see 22 . First, all free molecules are independently diffused along each dimension by generating normal random variables with variance $2 D_{S} \Delta t$. Next, potential reactions are evaluated to see whether they would have occurred during $\Delta t$. For bimolecular reactions, a binding radius $r_{B}$ is defined as how close the centers of two reactant molecules need to be at the end of $\Delta t$ in order to assume that the two molecules collided and bound during $\Delta t$. For unimolecular reactions, a random number is generated using the rate constant to declare whether the reaction occurred during $\Delta t$.

Particle-based methods tend to be less computationally efficient, but they do not have to meet the well-stirred requirement. Our system has an impulse of molecules being released into an environment with highly reactive enzymes (we will discuss specific rate constants in Section 5, but for now we note that we are generally interested in large $k_{1}$ ). A general criterion for subvolume size is that the typical diffusion time for each species should be much less than the typical reaction time; see [23. We cannot guarantee the satisfaction of this criterion for subvolume sizes that make physical sense (i.e., significantly larger than the size of individual molecules), so we adopt a particle-based method.

\subsection{Simulating Reactions}

Our bimolecular reaction (1) (the binding of $E$ and $A$ to form $E A$ ) is reversible, so we must be careful in our choice of binding radius $r_{B}$, time step $\Delta t$, and what we assume when $E A$ reverts back to $E$ and $A$ molecules. A relevant metric is the root mean square step length, $r_{r m s}$, between $E$ and $A$ molecules, given as [22, Eq. 23]

$$
r_{r m s}=\sqrt{2\left(D_{A}+D_{E}\right) \Delta t}
$$

If reaction (2) occurs, then the root mean square separation of the product molecules $A$ and $E$ along each dimension is $r_{r m s}$. Unless $r_{r m s} \gg r_{B}$, then these two reactants will likely undergo reaction (1) in the next time step. Generally, 
we need to define an unbinding radius specifying the initial separation of the $A$ and $E$ molecules when reaction (2) occurs. However, in the long time step limit, we can define $r_{B}$ as [22, Eq. 27]

$$
r_{B}=\left(\frac{3 k_{1} \Delta t}{4 \pi}\right)^{\frac{1}{3}},
$$

and this is valid only when $r_{r m s} \gg r_{B}$. Thus, if $r_{r m s}$ is much greater than $r_{B}$ found by (14), which we can impose by our selection of $k_{1}$ and $\Delta t$, then we do not need to implement an unbinding radius. Also, the evaluation of $r_{B}$ is much more involved when we are not in the long time step limit and requires generating a lookup table; see 22 for further details. We will select parameters so that $r_{r m s} \gg r_{B}$ is satisfied, even if $r_{r m s}$ becomes comparable with the size of the receiver, so we simply use (14).

We have a few additional comments on simulating reaction (11). It does not require the generation of any random values, besides those that are used to diffuse the individual molecules. However, we must check the position of every unbound $A$ molecule with that of every unbound $E$ molecule to see whether they are closer than $r_{B}$. For computational efficiency, we create subvolumes so that we only need to check the positions of enzymes in the current and adjacent subvolumes of the current free $A$ molecule. If we find a pair close enough, then we move both of them to the midpoint of the line between their centers and re-label them as a single $E A$ molecule.

Our two unimolecular reactions have the same reactant, $E A$, so we must consider both of them when calculating the probability of either reaction occuring. For (2), we have [22, Eq. 14]

$$
\operatorname{Pr}\{\text { Reaction (9) }\}=\frac{k_{-1}}{k_{-1}+k_{2}}\left[1-\exp \left(-\Delta t\left(k_{-1}+k_{2}\right)\right)\right],
$$

where $\operatorname{Pr}\{\cdot\}$ denotes probability and (3) has an analogous expression by switching $k_{-1}$ and $k_{2}$. A single random number uniformly distributed between 0 and 1 can then be used to determine whether a given $E A$ molecule reacts. If it does, then we place the products at the same coordinates.

\subsection{Simulating the Sender and Receiver}

When the sender releases an impulse of $N_{A} A$ molecules, we enforce an initial separation of $2 R_{A}$ between adjacent $A$ molecules, placing them in a spherical shape centered at the origin. At the receiver, we make observations at integer multiples of time step $\Delta t$. When an observation is made, all free $A$ molecules whose centres are within $V_{o b s}$ are counted.

\subsection{Selecting Component Parameters}

We now discuss practical parameter values for the underlying reaction-diffusion system. Specific enzymatic reactions, such as the breakdown of acetylcholine by 
acetylcholinesterase, are represented by specific molecules and are characterized by specific reaction rate constants. Most enzymes are proteins and are usually on the order of less than $10 \mathrm{~nm}$ in diameter; see [1, Ch. 4]. From (4), smaller molecules diffuse faster, so we are most likely to select small molecules as information molecules. Many common small organic molecules, such as glucose, amino acids, and nucleotides, are about $1 \mathrm{~nm}$ in diameter. In the limit, single covalent bonds between two atoms are about $0.15 \mathrm{~nm}$ long; see [1, Ch. 2].

Higher rate constants correlate to faster reactions. Bimolecular rate constants can be no greater than the collision frequency between the two reactants, i.e., every collision results in a reaction. The largest possible value of $k_{1}$ is on the order of $1.66 \times 10^{-19}$ molecule $\mathrm{e}^{-1} \mathrm{~m}^{3} \mathrm{~s}^{-1}$; see [15, Ch. 10] where the limiting rate is listed as on the order of $10^{8} \mathrm{~L} / \mathrm{mol} / \mathrm{s}$. $k_{2}$ usually varies between 1 and $10^{5} \mathrm{~s}^{-1}$, with values as high as $10^{7} \mathrm{~s}^{-1}$. In theory, we are not entirely limited to preexisting enzyme-substrate pairs; protein and ribozyme engineering techniques can be used to modify and optimize the enzyme reaction rate, specificity, or thermal stability, or modify enzyme function in the presence of solvents.

\section{Results and Discussion}

We are now prepared to present results comparing the observed number of $A$ molecules at a receiver with and without the presence of enzymes in the propagation environment. We assume that the environment has a viscosity of $10^{-3} \mathrm{~kg} \mathrm{~m}^{-1} \mathrm{~s}^{-1}$ and temperature of $25^{\circ} \mathrm{C}$. The sender emits $N_{A}=10^{4} \mathrm{~A}$ molecules, each having radius $R_{A}=0.5 \mathrm{~nm}$, in a single impulse. $V_{\text {enz }}$ is defined as a cube with side length $1 \mu \mathrm{m}$ and centered at the origin, so its size is on the order of a bacterial cell. $N_{E}=2 \times 10^{5} \mathrm{E}$ molecules having radius $R_{E}=2.5 \mathrm{~nm}$ are uniformly distributed throughout $V_{\text {enz }}$. For simplicity, we assume that $R_{E A}=R_{A}+R_{E}=3 \mathrm{~nm}$. In consideration of the limiting values of reaction rate constants, we choose $k_{1}=10^{-19}$ molecule ${ }^{-1} \mathrm{~m}^{3} \mathrm{~s}^{-1}, k_{-1}=10^{4} \mathrm{~s}^{-1}$, and $k_{2}=10^{6} \mathrm{~s}^{-1}$. We also set $\Delta t=0.5 \mu \mathrm{s}$, resulting in $r_{r m s}=22.9 \mathrm{~nm}$ and $r_{B}=2.28 \mathrm{~nm}$, so that $r_{r m s} \gg r_{B}$ is satisfied.

We compare the number of molecules observed at a receiver due to a single emission from the sender. In Fig. 3, we consider two receivers with radii $r_{o b s}=$ $\{25,45\} \mathrm{nm}$ and their centers placed at a distance of $\left|\overrightarrow{r_{0}}\right|=\{150,300\} \mathrm{nm}$ from the sender, respectively. The expected number of molecules is calculated using either (12) or (6) for enzymes present and absent, respectively. The observed number of $A$ molecules via simulation is averaged over at least 15000 independent emissions by the sender at $t=0$.

Let us first consider the receiver placed $150 \mathrm{~nm}$ from the sender. The maximum number of molecules is received about $8 \mu \mathrm{s}$ after emission. The maximum value is less than $15 \%$ higher in the absence of active enzymes; over 14 molecules are expected and observed on average via simulation without enzymes, compared to 12 molecules expected and 12.5 molecules observed with active enzymes. The decay from the maximum value is slower in the absence of active enzymes; $60 \mu \mathrm{s}$ after emission, 3 molecules are expected and observed without enzymes while 


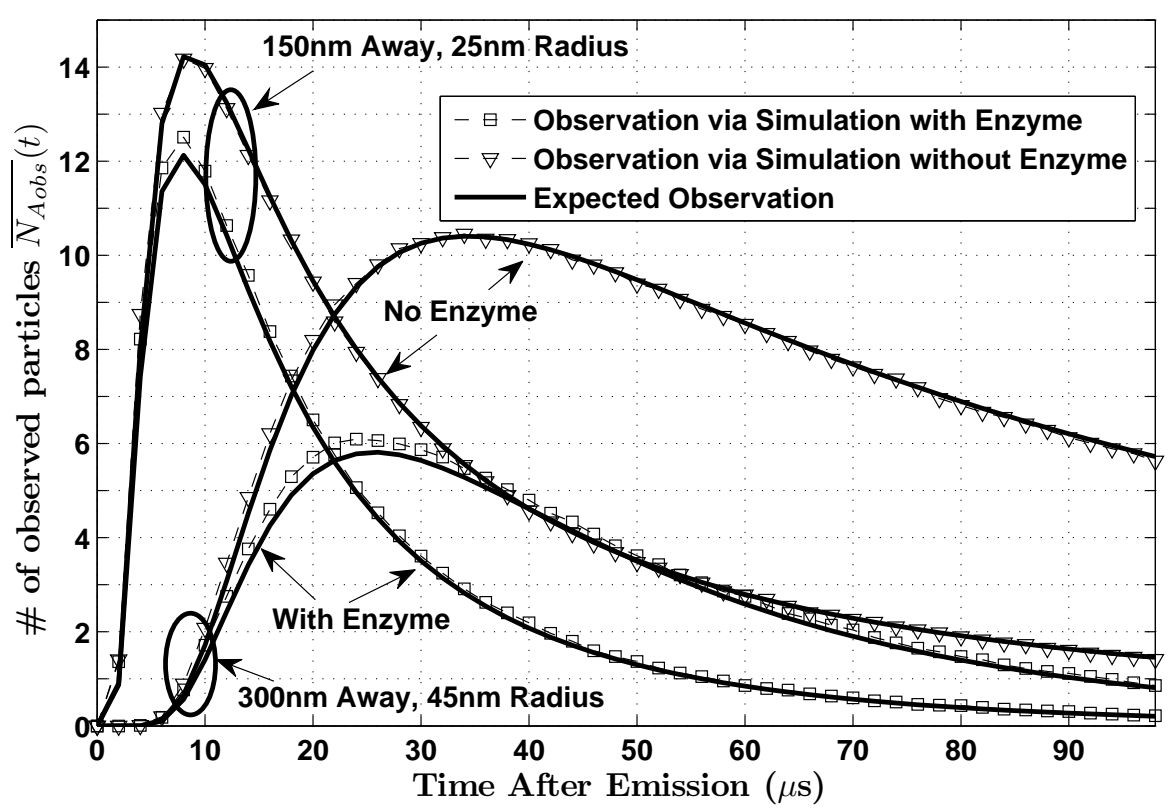

Fig. 3. Number of particles counted by receivers with radii $r_{o b s}=\{25,45\} \mathrm{nm}$ that are placed $\left|\overrightarrow{r_{0}}\right|=\{150,300\} \mathrm{nm}$ from the sender, respectively. The source releases $10^{4}$ molecules at $t=0$. Simulation and analytical results are shown both with and without active enzymes.

1 molecule is expected and observed with enzymes, a threefold difference. We see that, as previously noted, the expected number of observed $A$ molecules when active enzymes are present is a lower bound on the average number of $A$ molecules observed in simulation, and this is a relatively tight bound.

The simulation and analytical results for the receiver placed $300 \mathrm{~nm}$ from the sender follow the same general trends as those for the closer receiver, but with a few noteworthy differences. Obviously, the time elapsed before receiving the maximum number of molecules is greater and the maximum value is less than for the closer receiver, even though the receiver is larger (the receiver being larger accounts for how it is possible for this receiver to observe more molecules than the closer receiver after $23 \mu \mathrm{s}$ ). However, the change in the number of molecules received is much greater in the presence of active enzymes; the peak number of molecules is observed relatively sooner (about $25 \mu \mathrm{s}$ instead of about $35 \mu \mathrm{s}$ after emission), but the maximum number of molecules is less than $60 \%$ of that expected without enzymes (about 6 molecules instead of 10.5 molecules). Intuitively, being further from the sender gives more time for the $E$ molecules to bind to and then degrade the $A$ molecules.

Both receivers in Fig. 3 show that adding enzymes decreases the "tail" of diffusion while still providing a peak to be detected at the receiver. It is clear that the sender could emit impulses more often with less risk of ISI. For example, if the 
criterion for designing the bit interval $T_{B}$ was the time at which the expected number of particles is some fraction of the maximum expected number, then this time should be shorter in the presence of active enzymes. Alternatively, sender-receiver pairs could be placed closer together with less risk of co-channel interference. We leave formal proofs of these statements for future work, but they are intuitive given the results in Fig. 3 .

Finally, we consider in Fig. 4 the limiting case that we used to derive the bound (12), i.e., set $k_{2}=\infty, k_{-1}=0$, and co-locate all $A$ molecules at the origin when emitting. In this case, an $E$ molecule binding to an $A$ molecule immediately degrades the $A$ molecule while releasing the $E$ molecule, so all enzymes are always available to react. We otherwise maintain the same parameters that we used for Fig. 3. We see that the average number of particles observed via simulation with active enzymes agrees very well with that expected from (12), and that the average number of particles observed via simulation without active enzymes matches the value expected from (6), even though we are still assuming uniform $C_{A}$ throughout the receiver volume. This confirms that the looseness of the lower bound (12) in Fig. 3 comes from both the finite emission volume and the creation of $E A$ molecules. The slight looseness of the lower bound in Fig. 4 for the receiver $300 \mathrm{~nm}$ away and when enzymes are present is likely due to having a finite $V_{\text {enz }}$; some $A$ molecules are able to diffuse beyond $V_{e n z}$, where they cannot be degraded, and then enter the receiver volume after returning to $V_{\text {enz }}$. This effect is negligible at the receiver $150 \mathrm{~nm}$ away.

\section{Conclusions and Future Work}

In this paper, we introduced the concept of using enzymes in the propagation environment to improve the performance of a diffusive molecular communication system. Enzymes that break down information molecules are able to reduce the time that a sender must wait before being able to send additional information molecules. There is potential to increase the data rate and to decrease the probability of error. This gain in performance comes with no additional complexity required at either the sender or receiver.

The emphasis in this paper was the description of the underlying reactiondiffusion model and the selection of an appropriate simulation framework, thereby providing a foundation for performance analysis. On-going work includes the derivation of the bit error rate for this binary-coded communication network when multiple emissions are made by the sender given a bit interval $T_{B}$ and the reception scheme at the receiver. Furthermore, we are currently evaluating the analytical accuracy of the assumption that the concentration observed at the receiver is uniform. We must also consider the ability to choose reaction rate constants based on specific enzymes, as well as the enzyme concentration. In addition, dimensional analysis is useful to arbitrarily scale our system, compare different parameter sets, and derive the looseness of our receiver bound in terms of a dimensionless parameter. We also note that we could forego the use of enzymes altogether and use $A$ molecules with a faster natural degradation rate, 


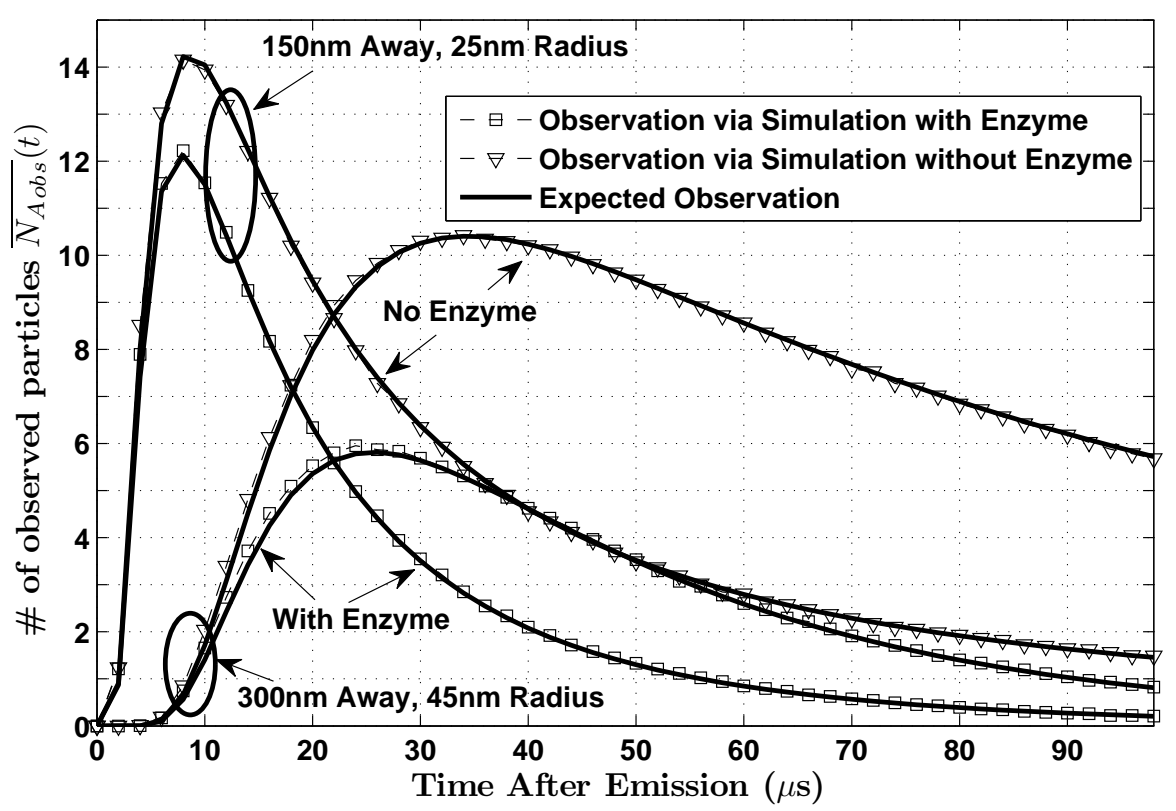

Fig. 4. Number of particles counted by receivers with radii $r_{o b s}=\{25,45\} \mathrm{nm}$ that are placed $\left|\overrightarrow{r_{0}}\right|=\{150,300\} \mathrm{nm}$ from the sender, respectively. The source releases $10^{4}$ molecules from a point at $t=0$. Simulation and analytical results are shown both with and without active enzymes, where $k_{2}=\infty$ and $k_{-1}=0$. The resultant difference between this figure and Fig. 3 is that here the curves generated via simulation are tighter to the curves generated by the analytical expressions.

as in [12, but without using the number of counted molecules as the amount of information received. This case would allow simpler and accurate analysis though we would have to be concerned with maintaining a stockpile of these molecules at the sender without them degrading before emission. Other relevant problems of interest include interference from nearby sender/receiver pairs and the potential mobility of the sender and receiver.

\section{References}

1. Alberts, B., Bray, D., Hopkin, K., Johnson, A., Lewis, J., Raff, M., Roberts, K., Walter, P.: Essential Cell Biology. 3rd edn. Garland Science (2010)

2. Akyildiz, I.F., Brunetti, F., Blazquez, C.: Nanonetworks: A new communication paradigm. Computer Networks 52(12) (May 2008) 2260-2279

3. Nakano, T., Moore, M.J., Wei, F., Vasilakos, A.V., Shuai, J.: Molecular communication and networking: Opportunities and challenges. IEEE Trans. Nanobiosci. 11(2) (Jun. 2012) 135-148

4. Nelson, P.: Biological Physics: Energy, Information, Life. Updated 1st edn. W. H. Freeman and Company (2008) 
5. Hiyama, S., Moritani, Y.: Molecular communication: Harnessing biochemical materials to engineer biomimetic communication systems. Nano Commun. Net. 1(1) (Mar. 2010) 20-30

6. Atakan, B., Akan, O.B.: Deterministic capacity of information flow in molecular nanonetworks. Nano Commun. Net. 1(1) (Mar. 2010) 31-42

7. Mahfuz, M.U., Makrakis, D., Mouftah, H.T.: Characterization of intersymbol interference in concentration-encoded unicast molecular communication. In: Proc. 2011 IEEE CCECE. (May 2011) 164-168

8. Einolghozati, A., Sardari, M., Beirami, A., Fekri, F.: Capacity of discrete molecular diffusion channels. In: Proc. 2011 IEEE ISIT. (Aug. 2011) 723-727

9. Chou, C.T.: Molecular circuits for decoding frequency coded signals in nanocommunication networks. Nano. Comm. Networks 3(1) (Mar. 2012) 46-56

10. Nakano, T., Okaie, Y., Vasilakos, A.V.: Throughput and efficiency of molecular communication between nanomachines. In: Proc. 2012 IEEE WCNC. (Apr. 2012) 704-708

11. Miorandi, D.: A stochastic model for molecular communications. Nano Commun. Net. 2(4) (Dec. 2011) 205-212

12. Moore, M.J., Suda, T., Oiwa, K.: Molecular communication: Modeling noise effects on information rate. IEEE Trans. Nanobiosci. 8(2) (Jun. 2009) 169-180

13. Naka, T., Shiba, K., Sakamoto, N.: A two-dimensional compartment model for the reaction-diffusion system of acetylcholine in the synaptic cleft at the neuromuscular junction. Biosystems 41(1) (Jan. 1997) 17-27

14. Cheng, Y., Suen, J.K., Radi, Z., Bond, S.D., Holst, M.J., McCammon, J.A.: Continuum simulations of acetylcholine diffusion with reaction-determined boundaries in neuromuscular junction models. Biophys. Chem. 127(3) (May 2007) 129-139

15. Chang, R.: Physical Chemistry for the Biosciences. University Science Books (2005)

16. Gillespie, D.T.: A rigorous derivation of the chemical master equation. Physica A 188(13) (Sep. 1992) 404-425

17. Pierobon, M., Akyildiz, I.F.: Information capacity of diffusion-based molecular communication in nanonetworks. In: Proc. 2011 IEEE INFOCOM 2011. (Apr. 2011) 506-510

18. Pierobon, M., Akyildiz, I.F.: A physical end-to-end model for molecular communication in nanonetworks. IEEE J. Sel. Areas Commun. 28(4) (May 2010) 602-611

19. Debnath, L.: Nonlinear Partial Differential Equations for Scientists and Engineers. 2nd edn. Birkhaeuser (2005)

20. Gillespie, D.T.: Stochastic simulation of chemical kinetics. Annu. Rev. Phys. Chem. 58(1) (May 2007) 35-55

21. Iyengar, K.A., Harris, L.A., Clancy, P.: Accurate implementation of leaping in space: the spatial partitioned-leaping algorithm. J. Chem. Phys. 132(9) (Mar. 2010) 094101

22. Andrews, S.S., Bray, D.: Stochastic simulation of chemical reactions with spatial resolution and single molecule detail. Physical Biology 1(3) (Aug. 2004) 137

23. Bernstein, D.: Simulating mesoscopic reaction-diffusion systems using the Gillespie algorithm. Phys. Rev. E 71(4) (Apr. 2005) 041103 